\title{
LOCAL INVARIANCE OF FREE TOPOLOGICAL GROUPS
}

\author{
by M. S. KHAN, SIDNEY A. MORRIS and PETER NICKOLAS
}

(Received 23rd July 1984)

\section{Introduction}

In 1948, M. I. Graev [2] proved that the free topological group on a completely regular Hausdorff space is Hausdorff, by showing that the free group admits a certain locally invariant Hausdorff group topology. It is natural to ask if Graev's locally invariant topology is the free topological group topology. If $X$ has the discrete topology, the answer is clearly in the affirmative. In 1973, Morris-Thompson [6] showed that if $X$ is not totally disconnected then the answer is negative. Nickolas [7] showed that this is also the case if $X$ has any (non-trivial) convergent sequence (for example, if $X$ is any non-discrete metric space). Recently, Fay and Smith Thomas handled the case when $X$ has a completely regular Hausdorff quotient space which has an infinite compact subspace (or more particularly a non-trivial convergent sequence). (Fay-Smith Thomas observe that their class of spaces includes some but not all those dealt with by Morris-Thompson.)

For convenience, we say a completely regular Hausdorff space is tolerable if it has a countably infinite family of closed subsets $A_{n}, n=1,2, \ldots$, such that $\bigcup_{n=1}^{\infty} A_{n}$ is not closed. We shall see that all the spaces above, considered by Morris-Thompson, Nickolas and Fay-Smith Thomas are tolerable. We prove that the free topological group on any tolerable space is not locally invariant.

Fay-Smith Thomas mention the space $X$ defined below as one for which the question of local invariance is undecided: Let $\mathbb{N}$ be the discrete space of natural numbers, and $X$ the subspace of the Stone-Cech compactification $\beta \mathbb{N}$ of $\mathbb{N}$ defined by $X=\mathbb{N} \cup\{p\}$, for any $p \in \beta \mathbb{N} \backslash \mathbb{N}$. Clearly $X$ is a tolerable space, and so the free topological group is not locally invariant.

We remind the reader that the Graev free topological group on a completely regular Hausdorff (pointed) space $X$ with basepoint $e$ is a topological group $F(X)$ which, algebraically, is a free group with $X \backslash\{e\}$ as a free basis, and is such that any continuous map from $X$ to a topological group, sending $e$ to the identity, extends uniquely to a continuous homomorphism on $F(X)$. The basepoint $e$ becomes the identity of $F(X)$, and the generating copy of $X$ has the topology of the original space. This construction is independent, up to isomorphism, of the choice of basepoint [2].

A topological group is said to be locally invariant if it has a basis at the identity of sets invariant under all inner automorphisms.

We need the following theorem.

Theorem A [2, 3]. Let $Y$ be any compact Hausdroff space. $A$ subset $C$ of $F(Y)$ is closed if and only if $C \cap F_{n}(Y)$ is compact, for all $n$, where $F_{n}(Y)$ is the set of all words in $F(Y)$ of reduced length at most $n$. 


\section{The Theorem}

Theorem. If $X$ is any tolerable space, then $F(X)$ is not locally invariant.

Proof. Let $A_{n}, n=1,2, \ldots$, be a family of closed subsets of $X$ such that $A=\bigcup_{n=1}^{\infty} A_{n}$ is not closed. Without loss of generality let the basepoint $e$ be in $\bar{A} \backslash A$. For each $n$, let $x_{n} \in A \backslash A_{n}$ (so $x_{n} \neq e$ ). For each $n$, let $B_{n}$ be the subset of $F(X)$ given by $B_{n}=x_{n}^{-n} A_{n} x_{n}^{n}$, and put $B=\bigcup_{n=1}^{\infty} B_{n}$. We shall prove that $B$ is closed in $F(X)$, but is not closed in any locally invariant topology on the underlying group of $F(X)$.

Let $\phi$ be the canonical embedding of $X$ in its Stone-Čech compactification $\beta X$. Then $\phi$ extends to a continuous homomorphism $\Phi: F(X) \rightarrow F(\beta X)$. Put

$$
C_{n}=\overline{\Phi\left(B_{n}\right)}=\Phi\left(x_{n}^{-n}\right) \overline{\Phi\left(A_{n}\right)} \Phi\left(x_{n}^{n}\right)
$$

and

$$
C=\bigcup_{n=1}^{\infty} C_{n}
$$

Clearly

$$
\begin{aligned}
\Phi^{-1}(C) & =\Phi^{-1}\left(\bigcup_{n=1}^{\infty} C_{n}\right) \\
& =\bigcup_{n=1}^{\infty} \Phi^{-1}\left(C_{n}\right) \\
& =\bigcup_{n=1}^{\infty} \Phi^{-1}\left(\Phi\left(x_{n}^{-n}\right) \overline{\Phi\left(A_{n}\right)} \Phi\left(x_{n}^{n}\right)\right) \\
& =\bigcup_{n=1}^{\infty} x_{n}^{-n} \Phi^{-1}\left(\overline{\Phi\left(A_{n}\right)}\right) x_{n}^{n} \\
& =\bigcup_{n=1}^{\infty} x_{n}^{-n} A_{n} x_{n}^{n},
\end{aligned}
$$

as $A_{n}$ is a closed subspace of $X$ and $\Phi$ is an embedding of $X$ into $\beta X$. So $\Phi^{-1}(C)=B$. But since the length of each word in $C_{m}$, for $m>n$, is $2 m+1>n, C \cap F_{n}(\beta X)=$ $\left(\bigcup_{i=1}^{n} C_{i}\right) \cap F_{n}(\beta X)$. So, since $\bigcup_{i=1}^{n} C_{i}$ is compact, $C \cap F_{n}(\beta X)$ is compact for each $n$, and therefore by Theorem A, $C$ is closed in $F(\beta X)$. Hence $B=\Phi^{-1}(C)$ is closed in $F(X)$.

Now let $\tau$ be any locally invariant group topology which induces the given topology on $X$. As $e \in \bar{A} \backslash A$ there exists a net $a_{\alpha}$ in $A$ such that $a_{\alpha} \rightarrow e$. Put $b_{\alpha}=x_{n}^{-n} a_{\alpha} x_{n}^{n}$, where $n=\min \left\{m: a_{\alpha} \in A_{m}\right\}$. Clearly $b_{\alpha} \rightarrow e$ in the locally invariant topology $\tau$. As $b_{\alpha} \in B$ but $e \notin B$, $B$ is not closed in $\tau$. This completes the proof.

Remark 1. The above theorem was announced without proof in [5]. It is interesting to note that the condition of tolerability is precisely that for which it is known that the Graev and Swierczkowski topologies [5] on the free group are distinct. 


\section{Applications}

We call a space intolerable if it is completely regular Hausdorff and is not tolerable. Of course all discrete spaces are intolerable. In Section 4 we give some examples of nondiscrete intolerable spaces.

Proposition 1. If $X$ is an intolerable space, then every countable subspace $Y$ of $X$ is closed in $X$ and its induced topology is discrete.

Proof. As $Y$ is the countable union of its singleton subsets, each of which is closed in $X, Y$ is closed in $X$. Similarly, if $y$ is any point of $Y$ then the countable set $Y \backslash\{y\}$ is closed in $X$. Hence $\{y\}$ is open in $Y$, and so $Y$ has the discrete topology.

The proof of the next proposition is trivial, and is therefore omitted.

Proposition 2. Every completely regular Hausdorff quotient space of an intolerable space is intolerable. Also, every subspace of an intolerable space is intolerable.

Corollary. Let $X$ be a completely regular Hausdorff space such that $X$ (or a completely regular Hausdorff quotient space of $X$ ) has any of the following properties:

(i) $X$ is a non-discrete metric space,

(ii) $X$ has a non-trivial convergent sequence,

(iii) $X$ has an infinite compact subspace,

(iv) $X$ has a non-discrete subspace which is a $k$-space,

(v) $X$ is not totally disconnected.

Then $F(X)$ is not locally invariant.

Proof. Clearly (i) $\Rightarrow$ (ii) $\Rightarrow$ (iii) $\Rightarrow$ (iv) $\Rightarrow$ (iii). By Proposition 1 , if $X$ satisfies (iii), then $X$ is tolerable, since otherwise the infinite compact Hausdorff subspace would necessarily contain a countably infinite closed discrete subspace, contradicting the compactness of the first subspace. If $X$ is not totally disconnected, then by the Lemma of [6], $X$ is tolerable. So if $X$ has any of the properties (i),..,(v), then $X$ is tolerable. Hence, by Proposition 2, if a completely regular Hausdorff space has a completely regular Hausdorff quotient space with any of the properties (i),..,(v), then $X$ is tolerable. Applying our Theorem of Section 2 then completes the proof.

Remark 2. The above Corollary contains the results of [1], [6] and [7].

Remark 3. We do not know whether the condition of being a tolerable space is necessary for $F(X)$ to be locally invariant. With this in mind, we end this section with a proposition which complements Proposition 2 and may be useful in later work.

Proposition 3. Let $X$ and $Y$ be completely regular Hausdorff spaces, with $Y$ a quotient space of $X$. If $F(X)$ is locally invariant, then $F(Y)$ is locally invariant.

Proof. If $\phi: X \rightarrow Y$ is a quotient map, then it is easily shown (and well known) that the canonical extension of $\phi$ to a continuous homomorphism $\Phi$ of $F(X)$ onto $F(Y)$ is a quotient map. Hence if $F(X)$ is locally invariant so too is $F(Y)$. 


\section{Examples}

In [1], Fay-Smith Thomas record an example, mentioned in Section 1, for which the problem of local invariance is not settled. The following Remark covers their example and more.

Remark 4. Let $X$ be any countably infinite completely regular Hausdorff space, and $\beta X$ its Stone-Cech compactification. If $X \subset Y \subseteq \beta X$, then $F(Y)$ is not locally invariant. This result follows from our Theorem, since $X$ is not closed in $Y$ which, by Proposition 1 , implies $Y$ is a tolerable space.

So far our only examples of intolerable spaces are the discrete spaces. We now introduce a family of non-discrete intolerable spaces, but first we observe that even any infinite product of discrete spaces (with at least 2 points) is tolerable. Indeed, any infinite product of non-trivial completely regular Hausdorff spaces is tolerable, since it has a subspace homeomorphic to the compact space formed by taking a countably infinite product of 2-point discrete spaces.

Example. Let $X$ be any set of cardinality $m>\aleph_{0}$. Further, let $e$ be any point of $X$ and $n$ any cardinal number satisfying $\aleph_{0} \leqq n<m$. We define a topology on $X$ by stating that the closed sets are all the subsets containing $e$, and all the subsets of cardinality $\leqq n$. It is easily seen that with this topology, $X$ is a non-discrete Hausdorff normal (and hence completely regular) topological space. Further, $X$ is intolerable.

So this family of examples is not covered by our Theorem, and we have been unable to establish whether or not each $F(X)$ is locally invariant.

Further intolerable spaces can be obtained by observing that all finite products, all box products, and all disjoint unions of intolerable spaces are intolerable.

Remark 5. In fact our proof of the Theorem in Section 2 yields more than we have said to date. Let $\tau_{s}$ be the induced topology (from $F(\beta X)$ ) on the free group $\Phi(F(X)$ ). It is shown in Nummela [8] that $\tau_{s}$ is the free topology (that is, $\Phi$ is an isomorphism of $F(X)$ onto $\Phi(F(X))$ if and only if $X$ is pseudocompact. So if $X$ is any nonpseudocompact tolerable space then $\tau_{s}$ is not the free topology, and the proof of the theorem shows that $\tau_{s}$ is not locally invariant.

Remark 6. Finally we point out that every intolerable space is pseudonormal. (Recall that a topological space $X$ is said to be pseudonormal if given any two disjoint closed subsets $A$ and $B$ such that one of them is countable, there exist disjoint open sets $U$ and $V$ such that $A \subseteq U$ and $B \subseteq V$. See [4].)

To see this let $X$ be intolerable and $A$ and $B$ as above. Without loss of generality, assume $A$ is countable. As $X$ is completely regular and Hausdorff, for each $a \in A$ there exists a continuous function $f_{a}: X \rightarrow[0,1]$ such that $f_{a}(a)=0$ and $f_{a}(B)=1$. Therefore, for each $a \in A$, there exist disjoint open sets $U_{a}$ and $V_{a}$ such that $a \in U_{a}$ and $B \subseteq V_{a}$. Then, since $X$ is intolerable $U=\bigcup_{a \in A} U_{a}$ and $V=\bigcap_{a \in A} V_{a}$ are disjoint open sets such that $A \subseteq U$ and $B \subseteq V$, which completes the proof. 


\section{REFERENCES}

1. Temple H. Fay and Barbara V. Smith Thomas, Free topological groups are almost never locally Invariant, Math. Colloq. Univ. Capetown 13 (1984), 1-8.

2. M. I. Graev, Free topological groups, Izvestiya Akad. Nauk SSSR. Ser. Mat. 12 (1948), 279-324. English trans.: Amer. Math. Soc. Transl. 35 (1951); reprint Amer. Math. Soc. Transl. (1) 8 (1962), 305-364.

3. John Mack, Sidney A. Morris and Edward T. Ordman, Free topological groups and the projective dimension of a locally compact abelian group, Proc. Amer. Math. Soc. 40 (1973), 303-308.

4. Arnold W. Miller On box products, Topology Applic. 14 (1982), 313-317.

5. Sidney A. Morris and Peter Nickolas, Locally Invariant topologies on free groups, Pacific J. Math. 103 (1982), 523-537.

6. Sidney A. Morris and H. B. Thompson, Invariant metrics on free topological groups, Bull. Austral. Math. Soc. 9 (1973), 83-88.

7. Peter Nickolas, Free topological groups and free products of topological groups (Ph.D. thesis, University of New South Wales, Australia, 1976).

8. E. Nummela, Uniform free topological groups and Samuel compactifications, Topology Applic. 13 (1982), 77-83.

Department of Mathematics

King Abdul Aziz University

P.O. Box 9028, ЈЕDDAH-21413,

Saudi Arabia

Department of Computing Science

UNIVERSITY OF WOLLONGONG

WOLLONGONG, 2500

Australia
Department of Mathematics

LA TROBE UNIVERSITY

BUNDOORA 3083

Australia 